\title{
Contribuições da educação interprofissional ao ensino odontológico no Brasil: uma revisão sistemática
}

Interprofessional education contributions to dental education in Brazil: a systematic review

Contribuciones de la educación interprofesional a la enseñanza dental en Brasil: una revisión sistemática

Karla Dias Castro Dias ${ }^{1 *}$, Diego dos Santos Dias ${ }^{2}$, Natália dos Santos Dias ${ }^{3}$, Maria Aparecida Turci.

\section{RESUMO}

Objetivo: Compreender a contribuição da Educação Interprofissional (EIP) ao ensino odontológico na formação dos odontólogos brasileiros. Métodos: Trata-se de uma revisão sistemática da literatura. A busca dos estudos foi conduzida por duas pesquisadoras, de forma independente, selecionando-se publicações dos últimos 10 anos, nos idiomas inglês, português e espanhol. Utilizaram-se as bases de dados da Biblioteca Virtual em Saúde (BVS) e os repositórios nacionais de dissertações e teses. Adicionalmente, realizou-se busca manual em anais de congressos nacionais de odontologia e em reuniões da Associação Brasileira de Ensino Odontológico (ABENO). Resultados: Encontraram-se majoritariamente relatos de experiência, exigindo maior evidência científica; o Programa de Educação pelo Trabalho para a Saúde (PET-Saúde) desempenha importante influência em EIP, aliando-se ao maior engajamento das Instituições de Ensino Superior (IES) públicas; as Unidades Básicas de Saúde (UBS) representam o cenário preferencial para prática da EIP. Os principais benefícios das experiências consistiram na troca de saberes, valorização de cada profissional, respeito entre os profissionais, desconstrução de estereótipos e despertamento de novas habilidades. Considerações Finais: A EIP parece contribuir positivamente na formação dos odontólogos. Verificou-se a necessidade de esforços contínuos da odontologia para investigação e aplicação da EIP no Brasil.

Palavras-chave: Ensino em odontologia, Educação interprofissional, Prática interprofissional.

\begin{abstract}
Objective: To understand the contribution of Interprofessional Education (IPE) among dentistry students in Brazil. Methods: This is a systematic review of the literature. The search was conducted by two researchers, independently, it was selected publications of the last 10 years in English, Portuguese and Spanish. It was used the following databases: Biblioteca Virtual em Saúde (BVS) and national repositories of dissertations and theses. In addition, manual search was conducted in national congresses proceedings and Associação Brasileira de Ensino Odontológico (ABENO) meetings. Results: The studies are mostly case reports, requiring greater scientific evidence; Programa de Educação pelo Trabalho para a Saúde (PET-Saúde) develops an important influence in IPE, there is a bigger involvement of public dentistry schools than private; Unidades Básicas de Saúde (UBS) represent the preferential scenario for the practice of IPE. The main benefits of the experiences consisted in the sharing of learning, appreciation of each professional, respect among professionals, deconstruction of stereotypes and awakening of new skills. Final Consideration: IPE seems to
\end{abstract}

1 Universidade José do Rosário Vellano (UNIFENAS), Belo Horizonte - MG.

*E-mail: karladiascastro@yahoo.com.br

2 Universidade Estadual de Montes Claros (UNIMONTES), Montes Claros - MG.

${ }^{3}$ Centro Universitário UniFG (UNIFG), Guanambi - BA.

SUBMETIDO EM: 1/2021

ACEITO EM: 2/2020

PUBLICADO EM: 2/2021 
contribute positively to the dentist's graduation. This study verified a need for continued efforts by dental community to investigate and apply IPE in Brazil.

Key words: Dentistry education, Interprofessional education, Interprofessional practice.

\section{RESUMEN}

Objetivo: Comprender la contribución de la educación interprofesional (EIP) a los estudiantes de odontología en Brasil. Métodos: Se trata de una revisión sistemática de la literatura. La búsqueda fue realizada por dos investigadores, independientemente. Fueran seleccionadas publicaciones de los últimos 10 años en inglés, portugués y español. Fueran utilizadas las bases de datos de la Biblioteca Virtual em Saúde (BVS) y repositorios nacionales de disertaciones y tesis. Además, se realizó búsqueda manual en actas de congresos nacionales de odontología y reuniones de la Associação Brasileira de Ensino Odontológico (ABENO).

Resultados: Los estudios son en su mayoría reporte de casos, que requieren mayor evidencia científica; el Programa de Educação pelo Trabalho para Saúde (PET-Saúde) desarrolla influencia importante en la EIP, hay mayor participación de escuelas públicas de odontología que de las privadas; Unidades Básicas de Saúde (UBS) representan el escenario preferencial para EIP. Los principales beneficios de las experiencias: intercambio de aprendizajes, aprecio de cada profesional, respeto entre los profesionales, deconstrucción de estereotipos y despertar de nuevas habilidades. Consideraciones Finales: EIP parece contribuir positivamente a la graduación del odontólogo. Este estudio verificó la necesidad de esfuerzos continuos para aplicar EIP en Brasil.

Palabras clave: Enseñanza dental, Educación interprofesional, Práctica interprofesional.

\section{INTRODUÇÃO}

O ensino da odontologia tem se modificado de forma significativa nos últimos anos. A inserção da interprofissionalidade tem promovido um relevante impacto no ensino da odontologia moderna. As práticas colaborativas têm proporcionado maior integralidade, assegurando a otimização dos cuidados em saúde (ANDREWS EA, 2017; SARAIVA AM, et al., 2018).

Estudos apontam que nos próximos quarenta anos, a população irá requerer cuidados odontológicos distintos, que estarão intimamente relacionados à interprofissionalidade. Os cuidados restauradores e protéticos serão diminuídos, ao passo que exigir-se-ão cuidados mais complexos, relacionados às doenças sistêmicas e suas implicações. Nesse sentido, os profissionais da odontologia necessitam estar preparados para lidar com as condições sistêmicas de saúde dos pacientes, sendo fundamental a Educação Interprofissional (EIP) nesse processo de formação (WEINTRAUB JA, 2017).

No cenário atual, diante da pandemia Coronavirus Disease 2019 (COVID-19), o cirurgião-dentista, assim como os demais profissionais da saúde são desafiados a articularem uma sólida força de trabalho, fundamentada na interprofissionalidade e nas práticas colaborativas para superação dos problemas de saúde (SOUZA SV e ROSSIT RAS, 2020).

O termo Educação Interprofissional (EIP) vem sendo utilizado ao longo dos últimos 40 anos (ANDREWS EA, 2017). A EIP é caracterizada pelo compartilhamento de aprendizado entre estudantes de duas ou mais profissões diferentes, que resulta na melhoria da condição de saúde da população (ANDREWS EA, 2017; HAMIL LM, 2017; TOMPSEN NN, et al., 2018; REEVES S, 2016).

O conceito da interprofissionalidade se destaca por trazer uma proposta diferenciada. Não se trata apenas de um trabalho intraprofissional, em que profissionais de uma mesma área trabalham num mesmo ambiente com funções diferentes, como por exemplo: cirurgiões-dentistas, técnicos em saúde bucal, auxiliar de saúde bucal (HAMIL LM, 2017).

A EIP extrapola também o conceito da multiprofissionalidade, em que distintos profissionais estão presentes num só ambiente, mas não necessariamente atuando juntos (ARAÚJO TAM, et al., 2017). Pode- 
se ainda diferenciar a EIP do conceito de interdisciplinaridade que se refere à integração de saberes (FARIAS DN, et al., 2018).

A interprofissionalidade refere-se, portanto, a uma troca de conhecimento entre profissionais de áreas distintas, que passam a adquirir novos conhecimentos que não se restringem apenas à sua área específica de atuação e, assim, dividem as responsabilidades e decisões entre si (HAMIL LM, 2017).

Atualmente, no Brasil há 412 escolas de odontologia (CONSELHO FEDERAL DE ODONTOLOGIA, 2019). Algumas dessas escolas já relatam experiências com EIP, sua aceitação pelos acadêmicos e sua importância na formação dos odontólogos brasileiros (TOMPSEN NN, et al., 2018).

As universidades devem assegurar a formação de profissionais capazes de garantir aos pacientes o princípio da integralidade previsto pelo Sistema Único de Saúde (SUS), que está estreitamente relacionado à EIP. O indivíduo deve ser analisado de forma integral, seu contexto social deve ser levado em consideração para que as demandas do indivíduo sejam integralmente sanadas. Para tanto, são necessários profissionais generalistas, humanistas, com crítica reflexiva e com flexibilidade para continuar aprendendo e assimilando novos conhecimentos ao longo da sua carreira (ROCHA NB, et al., 2017).

Diante disso, faz-se necessário o aprofundamento das discussões e estudos dessa temática, EIP, entre as instituições de ensino, professores e sociedade civil, para que mudanças sejam implantadas e uma população bem cuidada seja o reflexo de tais mudanças (LIMA AFS, et al., 2019).

Portanto, perante as necessidades observadas, o objetivo deste estudo é compreender a contribuição da EIP ao ensino odontológico na formação dos odontólogos das escolas brasileiras.

\section{MÉTODOS}

Foi realizada uma revisão sistemática da literatura em busca de estudos que abordassem relatos sobre a temática EIP nas escolas de odontologia brasileiras. Para a busca dos estudos foi desenvolvido um Protocolo de Revisão Sistemática cadastrado na plataforma The Open Science Framework (OSF) (OSF, 2020).

Uma revisão sistemática é nomeada qualitativa quando a integração de estudos primários não é combinada estatisticamente por meio de uma metanálise. Portanto, a análise é desenvolvida a partir de uma observação ampla e complexa com uma abordagem interpretativa e naturalística do mundo (ALENCAR ES e ALMOULOUD SA, 2017).

A pergunta de revisão é fundamental para a condução de uma boa revisão sistemática, devendo ser estruturada de forma clara e objetiva para contemplar um resultado com o mínimo de viés possível. No caso de revisões sistemáticas qualitativas a pergunta deve ser estruturada com os seguintes elementos, que constituem o acrônimo population/ outcome/ type of study (POT): população ou alvo da pesquisa (P), desfecho (O) e tipo de estudo (T) (GALVÃO TF, et al., 2015; DE-LA-TORRE-UGARTE-GUANILO MC, et al., 2011).

Neste estudo a pergunta de revisão é: "A EIP contribui positivamente na formação dos cirurgiõesdentistas?" Tem-se, neste caso, o ensino interprofissional na odontologia como alvo da pesquisa (P); as descrições das experiências vivenciadas pelos alunos são o desfecho (O); e as pesquisas qualitativas, observacionais e comparativas são os tipos de estudos (T).

Dentre os tipos de estudo foram incluídos: relatos de experiência, relatos de caso e estudos experimentais que apresentassem quaisquer tipos de intervenções associadas ao tema da revisão, descritas de forma clara e objetiva. Foram excluídas as revisões narrativas que não apresentaram relato de experiência e os estudos que não abordaram alunos da graduação da odontologia.

A busca dos estudos primários foi realizada nos idiomas inglês, português e espanhol, nas seguintes bases de dados: Literatura Latino Americana e do Caribe em Ciências da Saúde (Lilacs), Scientific Electronic Library Online (Scielo), Biblioteca Brasileira de Odontologia (BBO) e Medical Literature Analysis and Retrieval System Online (MEDLINE) através da Biblioteca Virtual em Saúde (BVS). 
Para garantir que nenhum estudo importante deixasse de ser rastreado, foi desenvolvida uma busca isolada na BBO e na Rede Unida. A pesquisa também foi feita em repositórios de dissertações e teses: Portal de Dissertações e Teses da Capes e na Biblioteca Digital Brasileira de Teses e Dissertações.

Adicionalmente, foi conduzida uma busca manual em anais de congressos das escolas de odontologia nacionais e reuniões da Associação Brasileira de Ensino Odontológico (ABENO).

Os estudos foram buscados nas bases citadas utilizando-se os seguintes descritores: interprofessional education/ educação interprofissional/ educación interprofesional, formação interprofissional, interprofessional collaborative practice/ prática interprofissional colaborativa/ práctica interprofesional colaborativa, interprofessional relationships/ relações interprofissionais/ relaciones interprofesionales, interprofessional collaboration/ colaboração interprofissional/ colaboración interprofesional, interprofessional teamwork/ trabalho em equipe, dental teaching/ ensino em odontologia/ enseñanza dental.

Foram utilizados filtros de data da publicação, restringindo-se aos os últimos 10 anos, a fim de garantir que a questão abordada esteja de acordo com a realidade atual das escolas de odontologia brasieiras.

Os resultados das buscas foram exportados para o aplicativo Rayyan QCRI, no qual se realizou a seleção das referências (OUZZANI M, et al., 2016).

Os artigos identificados pela estratégia de busca inicial foram avaliados de forma independente pelas duas revisoras utilizando-se a aplicativo Rayyan QCRI em modo blind on. Antes de iniciar a seleção, foram identificadas e excluídas as duplicatas.

O rastreamento dos estudos foi feito pela leitura dos títulos e resumos e os estudos selecionados por pelo menos uma das revisoras foi levado para a próxima etapa. Os estudos foram marcados como "incluído" ou "excluído" no aplicativo e duas novas listas de estudos foram emitidas no software Excel, selecionando-se os estudos "incluídos" e "em conflito" para nova análise.

A análise de elegibilidade foi realizada pela leitura integral dos estudos de forma independente pelas duas revisoras. Nessa etapa, os motivos de exclusão foram registrados e a discordância entre as autoras foi resolvida em reunião de consenso.

O processo de seleção foi registrado com detalhes suficientes para completar um fluxograma Preferred Reporting Items for Systematic Reviews and Meta-Analyses (PRISMA) (GALVÃO TF, et al., 2015).

Para extração dos dados foram utilizados formulários padronizados em planilha do software Excel, sendo coletados dados relativos a: referência bibliográfica, método, objetivo, descrição do estudo, descrição da experiência, componentes essenciais da experiência, resultados e coleta de dados, aspectos que competem para o êxito, contexto e circunstâncias, conclusões, influência no ensino. Os dados foram sintetizados de forma qualitativa, gerando o relatório de revisão.

Os estudos selecionados foram avaliados quanto a qualidade, utilizando-se uma ferramenta adaptada, apresentada no estudo de Maimares W, et al. (2013). Trata-se de checklist utilizado em revisões sistemáticas que abordam um conjunto de estudos qualitativos e quantitativos.

\section{RESULTADOS E DISCUSSÂO}

A busca realizada na BVS, BBO, Rede Unida, Portal de Dissertações e Teses da Capes e na Biblioteca Digital Brasileira de Teses e Dissertações resultou em 535 estudos.

A busca manual em outras fontes resultou em 12 estudos. Após descartar cinco duplicados, obteve-se um total de 542 estudos. Através da leitura dos títulos e resumos foram excluídos 498 estudos, compondo uma relação de 44 estudos elegíveis após consenso entre as pesquisadoras.

Em seguida, foi realizada a leitura dos textos completos para aplicação dos critérios previamente definidos e, após consenso das duas pesquisadoras, 29 estudos foram excluídos restando 15 estudos para a síntese qualitativa. 
O processo de seleção, constituído pelas diferentes fases desta revisão sistemática e o quantitativo de estudos selecionados em cada etapa, foi registrado em um fluxograma PRISMA (Figura 1).

Figura 1 - Fluxograma PRISMA: fases da revisão sistemática e quantitativo de estudos selecionados.

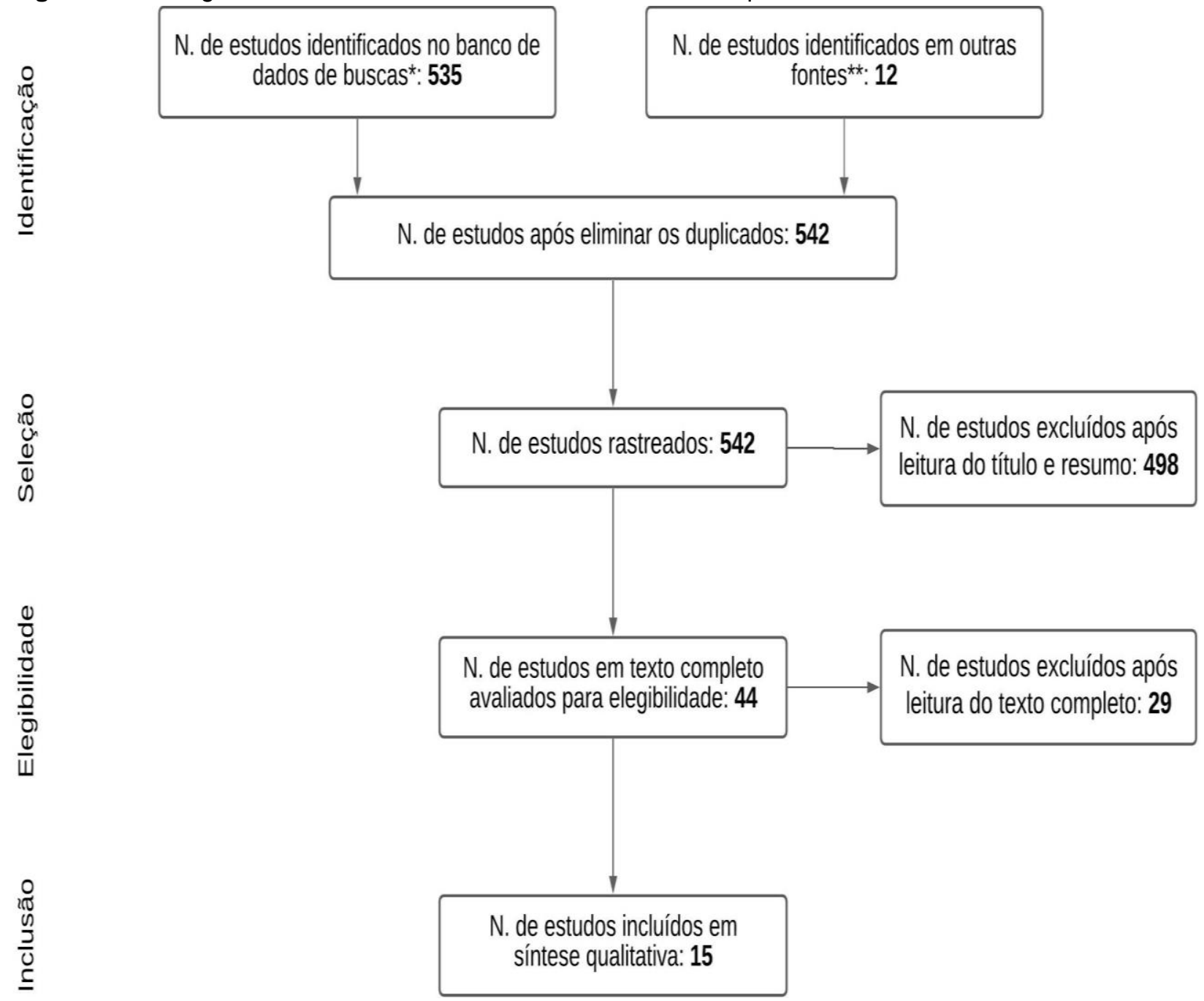

Legenda: Preferred Reporting Items for Systematic Reviews and Meta-Analyses (PRISMA).

Fonte: Dias KDC, et al., 2021.

As experiências contidas nesses estudos permitiram a análise das práticas de EIP na odontologia segundo proposto neste trabalho (Quadro 1). 
Quadro 1 - Descrição das experiências em EIP e conclusões dos estudos selecionados.

\begin{tabular}{|c|c|c|c|}
\hline $\mathbf{N}$ & Autores (Ano) & Descrição da experiência & Conclusão do estudo \\
\hline 1 & $\begin{array}{l}\text { CASSIANO CCZ } \\
(2016)\end{array}$ & $\begin{array}{c}\text { Observação participante do trabalho de campo registrada in loco } \\
\text { seguida de entrevistas com os preceptores dos estágios em } \\
\text { Estágio em Odontologia Coletiva e do PET-Saúde. Acadêmicos de } \\
\text { odontologia, biomedicina, farmácia, nutrição, medicina, } \\
\text { enfermagem e educação física realizaram: aplicação de } \\
\text { questionário aos membros da ESF, roda de conversa sobre } \\
\text { bullying com estudantes de } 10 \text { a } 11 \text { anos, entrega de folder sobre } \\
\text { câncer de boca e exame clínico, visita domiciliar com o mesmo } \\
\text { tema e apresentação das atividades desenvolvidas no estágio } \\
\text { para equipe da ESF. }\end{array}$ & $\begin{array}{c}\text { A aproximação ensino-serviço colabora para a formação dos } \\
\text { futuros profissionais e para atualização dos preceptores, além de } \\
\text { beneficiar a comunidade. A presença dos estagiários é um fator } \\
\text { motivacional para as equipes, com troca de informações entre } \\
\text { estagiários, preceptores e docentes. }\end{array}$ \\
\hline 2 & $\begin{array}{l}\text { CAVALCANTI MS, et } \\
\text { al. (2019) }\end{array}$ & $\begin{array}{c}\text { Estudantes de odontologia, medicina e enfermagem realizaram } \\
\text { escovação supervisionada com } 50 \text { escolares, houve } \\
\text { demonstração em modelo e distribuição de creme dental e } \\
\text { escova. Os acadêmicos trabalharam juntos sob a supervisão da } \\
\text { preceptora dentista. }\end{array}$ & $\begin{array}{c}\text { Os pré-escolares reproduziram o que foi aprendido, tornando-se } \\
\text { capazes de realizarem a higienização bucal correta. A atividade } \\
\text { mostrou-se relevante entre os acadêmicos e a comunidade, } \\
\text { permitindo a prática interprofissional, valorizando o cuidado, } \\
\text { promovendo a saúde e prevenindo agravos. }\end{array}$ \\
\hline 3 & $\begin{array}{l}\text { FERRAZ AMLJ, et al. } \\
\qquad(2014)\end{array}$ & $\begin{array}{c}\text { Implantação de um Projeto Pedagógico Interprofissional com } \\
\text { estudantes dos cursos de Enfermagem, Farmácia, Fisioterapia e } \\
\text { Odontologia. }\end{array}$ & $\begin{array}{l}\text { Identificou-se: incorporação do conceito de formação e trabalho } \\
\text { interprofissional; efetivação do trabalho em equipe; discussão de } \\
\text { papéis profissionais; respeito às especificidades de cada curso, } \\
\text { por meio da construção da identidade profissional; compromisso } \\
\text { na solução de problemas; e negociação na tomada de decisão. }\end{array}$ \\
\hline 4 & $\begin{array}{l}\text { FONSECA GS, et al. } \\
\qquad(2017)\end{array}$ & $\begin{array}{l}\text { Pesquisadora e acadêmico de odontologia realizaram observação } \\
\text { participante das atividades realizadas na UBS: acompanhamento } \\
\text { das consultas clínicas, vivência na organização do trabalho na } \\
\text { UBS e interação com a equipe de saúde. }\end{array}$ & $\begin{array}{c}\text { A experiência de Educação Interprofissional vivenciada reforçou o } \\
\text { potencial pedagógico no sentido de transformar o modelo de } \\
\text { formação fragmentado, ainda vigente nas faculdades de } \\
\text { odontologia. }\end{array}$ \\
\hline 5 & $\begin{array}{c}\text { FRAGA AT e } \\
\text { GHISLENI AP (2019) }\end{array}$ & $\begin{array}{c}\text { Alunos e professores dos cursos de fisioterapia, medicina, } \\
\text { odontologia, fonoaudiologia, nutrição, educação física e } \\
\text { enfermagem se reuniam para discutir as interfaces e conexões } \\
\text { das profissões na gerontologia. Foi elaborado um instrumento de } \\
\text { avaliação multidimensional da pessoa idosa. }\end{array}$ & $\begin{array}{l}\text { O projeto de extensão mostrou-se fundamental para repensar o } \\
\text { formato de ensino das graduações em saúde. A desconstrução } \\
\text { de estereótipos apresentou-se como resultado da interação entre } \\
\text { os alunos e professores dos diferentes cursos. }\end{array}$ \\
\hline
\end{tabular}




\begin{tabular}{|c|c|c|c|}
\hline $\mathbf{N}$ & Autores (Ano) & Descrição da experiência & Conclusão do estudo \\
\hline 6 & FUKUI VI (2018) & $\begin{array}{l}\text { Foi realizada avaliação dos docentes, preceptores e estudantes da } \\
\text { disciplina Prática Multiprofissional em Atenção Básica à Saúde. } \\
\text { Elegeram-se sete categorias: aprendizagem, experiência, } \\
\text { universidade, cenário de prática, cuidado, processo de trabalho } \\
\text { em saúde e SUS. }\end{array}$ & $\begin{array}{l}\text { Estudantes e preceptores concordaram que a aprendizagem e a } \\
\text { busca pelo conhecimento devem partir de questões que os } \\
\text { interessem e os envolvam. O cenário de prática apresentou-se } \\
\text { como um ambiente gerador de novos desafios e serviu para } \\
\text { concretizar ou questionar conceitos teóricos. }\end{array}$ \\
\hline 7 & $\begin{array}{l}\text { LIMA CR et al. } \\
\qquad(2019)\end{array}$ & $\begin{array}{c}\text { Estudantes do PET-Saúde aplicaram questionário à equipe da } \\
\text { ESF sobre o tema interprofissionalidade. Em seguida, realizaram } \\
\text { uma oficina com eles sobre o tema. }\end{array}$ & $\begin{array}{l}\text { A experiência revelou a importância de ampliar os conhecimentos } \\
\text { das equipes de saúde da família sobre o tema, tendo em vista } \\
\text { que a eficiência dos atendimentos realizada sob a ótica da } \\
\text { interprofissionalidade e colaboração representam um avanço na } \\
\text { organização do trabalho e na qualidade da assistência em saúde. }\end{array}$ \\
\hline 8 & $\begin{array}{l}\text { NORONHA FS, et al. } \\
(2019)\end{array}$ & $\begin{array}{l}\text { Estudantes do PET-Saúde realizaram oficina com a equipe da } \\
\text { ESF Antônio Pimenta para destacar a importância da } \\
\text { interprofissionalidade, desenvolvendo a conscientização dos } \\
\text { usuários por meio de distribuição de história em quadrinhos. }\end{array}$ & $\begin{array}{l}\text { A interprofissionalidade na Atenção Primária à Saúde mostrou o } \\
\text { potencial de trazer diversos ganhos para o sistema de saúde, } \\
\text { profissionais e usuários. O PET-Saúde apresentou papel } \\
\text { essencial para o seu desenvolvimento. }\end{array}$ \\
\hline 9 & $\begin{array}{l}\text { OLIVEIRA ML, et al. } \\
\text { (2019) }\end{array}$ & $\begin{array}{l}\text { Oficina direcionada à equipe da ESF Maracanã para incorporar a } \\
\text { prática da interprofissionalidade. }\end{array}$ & $\begin{array}{l}\text { O PET-Saúde/Interprofissionalidade revelou uma oportunidade } \\
\text { para despertar e desenvolver habilidades e competências durante } \\
\text { a formação, possibilitando reflexão, além de oferta conjunta de } \\
\text { atendimento humanizado, completo e eficaz em saúde à } \\
\text { comunidade. }\end{array}$ \\
\hline 10 & PEREIRA PM (2016) & $\begin{array}{l}\text { Alunos, tutores e preceptores do programa PET-Saúde têm } \\
\text { oportunidade de vivenciar a experiência IP através de pesquisa } \\
\text { bibliográfica sobre o tema, participação em atividades de } \\
\text { integração (grupos de discussão, seminários, eventos, elaboração } \\
\text { de pesquisas), atuação nos serviços de saúde com intervenção na } \\
\text { comunidade (projetos educativos e promoção de saúde com } \\
\text { estudantes de outras profissões sob a supervisão de preceptores } \\
\text { das mais diversas áreas da saúde). }\end{array}$ & $\begin{array}{l}\text { A experiência despertou um olhar diferenciado aos demais } \\
\text { profissionais, respeitando e reconhecendo o papel de cada um } \\
\text { para a valorização do SUS e seu funcionamento, estimulando o } \\
\text { desejo de atender as necessidades da população. }\end{array}$ \\
\hline
\end{tabular}




\begin{tabular}{|c|c|c|c|}
\hline $\mathbf{N}$ & Autores (Ano) & Descrição da experiência & Conclusão do estudo \\
\hline 11 & $\begin{array}{l}\text { ROCHA NB, et al. } \\
\qquad(2017)\end{array}$ & $\begin{array}{l}\text { Reflexões sobre a participação na disciplina Atenção a Saúde com } \\
\text { acadêmicos de biomedicina, educação física, farmácia, psicologia, } \\
\text { odontologia, enfermagem e medicina. Uma disciplina com } \\
\text { influência da EIP visando integrar os alunos das diversas áreas da } \\
\text { saúde, conduzindo grupos interprofissionais para atuar em estágio } \\
\text { na comunidade na UBS e vivenciar relações IP. }\end{array}$ & $\begin{array}{c}\text { Através da experiência, os estudantes relataram: ótima interação } \\
\text { interprofissional (23\%), contato com paciente }(20 \%) \text {, visitas } \\
\text { domiciliares que marcaram a vida pessoal e profissional }(20 \%) \text { e } \\
\text { visão da estrutura da UBS (20\%). A maioria dos estudantes (80\%) } \\
\text { aprovou o tutor. }\end{array}$ \\
\hline 12 & $\begin{array}{l}\text { SARAIVA AM, et al. } \\
\qquad(2018)\end{array}$ & $\begin{array}{c}\text { Acadêmicos do } 1^{0} \text { ano de odontologia participaram da disciplina } \\
\text { de atenção em saúde com acadêmicos de Medicina, Farmácia, } \\
\text { Biomedicina, Educação Física, Psicologia e Enfermagem e } \\
\text { relatam em portfólio suas experiências. }\end{array}$ & $\begin{array}{l}\text { A experiência demonstrou a importância do trabalho em ambiente } \\
\text { interprofissional, das metodologias ativas para o aprendizado dos } \\
\text { alunos, do contato com o SUS para o conhecimento e a postura } \\
\text { dos alunos em realização à disciplina destacando que obtiveram } \\
\text { conhecimentos com os colegas de outros cursos que } \\
\text { possivelmente não teriam se não estivessem no contexto da EIP. }\end{array}$ \\
\hline 13 & $\begin{array}{l}\text { SILVA MLO, et al. } \\
\qquad(2019)\end{array}$ & $\begin{array}{l}\text { Oficina direcionada aos usuários da ESF Maracanã para abordar o } \\
\text { tema interprofissionalidade através de teatro. }\end{array}$ & $\begin{array}{c}\text { A atividade possibilitou o exercício da interprofissionalidade na } \\
\text { prática do atendimento, caracterizando-se como agregadora e } \\
\text { fortalecedora da assistência. }\end{array}$ \\
\hline 14 & $\begin{array}{l}\text { SOUZA RMR, et al. } \\
\qquad(2013)\end{array}$ & $\begin{array}{c}\text { Acadêmica de odontologia e enfermeira realizaram uma atividade } \\
\text { com mães de crianças até } 3 \text { anos na UBS Nova Conquista em } \\
\text { João Pessoa. Foi desenvolvida uma roda de conversa sobre a } \\
\text { higienização bucal de bebês e em seguida foi realizada a consulta } \\
\text { de puericultura. }\end{array}$ & $\begin{array}{c}\text { Permitiu-se a integração dos conhecimentos de enfermagem e } \\
\text { odontologia para promoção de saúde da população, trazendo } \\
\text { uma percepção de que a saúde bucal é indissociável da saúde } \\
\text { geral. }\end{array}$ \\
\hline 15 & $\begin{array}{l}\text { ZAJKOWSKI LA, et } \\
\text { al. (2017) }\end{array}$ & $\begin{array}{l}\text { Acadêmicos de odontologia realizaram observação de idosa que } \\
\text { tinha dificuldade de interação e estava sempre isolada. Após } \\
\text { aproximação, verificou-se a prótese em más condições de } \\
\text { higienização, associada a dificuldades de memorização das } \\
\text { instruções de higiene. Após observação foi realizada intervenção } \\
\text { por acadêmicos de odontologia e terapia ocupacional. }\end{array}$ & $\begin{array}{l}\text { A abordagem interdisciplinar agregou conhecimentos e ideias ao } \\
\text { caso, permitindo resgatar saúde e bem-estar. }\end{array}$ \\
\hline
\end{tabular}

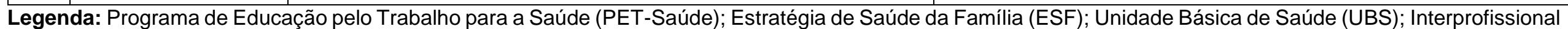
(IP); Educação Interprofissional (EIP). Fonte: Dias KDC, et al., 2021. 
Baseando-se na ferramenta de avaliação da qualidade de estudos qualitativos mostrada por Maimares W, et al. (2013) tivemos o seguinte resultado: seis estudos tiveram a resposta "sim" para as perguntas da ferramenta utilizada, nove estudos tiveram algumas das respostas "não". As perguntas são sobre os seguintes aspectos: fundamentação teórica, objetivos do estudo, contexto, amostra, descrição da coleta e análise de dados, confiabilidade da análise de dados, clareza sobre as conclusões tiradas, reflexividade, generalizabilidade e ética.

Dos quinze estudos incluídos, dois são dissertações de mestrado, quatro são artigos científicos e nove são resumos publicados em congressos e reuniões da ABENO. Dessa forma, os nove estudos têm tamanhos limitados e, portanto, não conseguem contemplar todas as perguntas da ferramenta. Foi de suma importância, trazer esses trabalhos, ainda que com menor evidência científica, devido ao número reduzido de estudos encontrados e com o objetivo de se esgotar as experiências constatadas na literatura nesta revisão sistemática.

Os resultados e suas interpretações serão apresentados em diferentes categorias: métodos e instrumentos de medidas, tipos de experiências, distribuição dos estudos por Instituição de Ensino Superior (IES): localização e categoria pública ou privada, cenário da prática, aspectos que competem para o êxito da experiência e influência da experiência no ensino.

Pode-se observar que a maioria dos estudos incluídos se trata de relatos de experiência. Dos quinze estudos avaliados, onze são relatos de experiência. Segundo Parente RCM, et al. (2012) os relatos de experiência foram durante muito tempo a única fonte de informações científica na área da saúde, através deles técnicas eram incorporadas à prática clínica rotineiramente. Entretanto, com o surgimento da medicina baseada em evidências este tipo de estudo tornou-se cada vez menos utilizado para esse fim devido à simplicidade da metodologia envolvida. Os instrumentos de medida utilizados foram em sua maioria: a mudança de hábito e incorporação do conhecimento, correspondendo a $60 \%$. Entretanto, a mensuração dessas variáveis é de caráter qualitativo.

Diante do exposto, há que se ressaltar a existência da Escala Jefferson de Atitudes Relacionadas à Colaboração Interprofissional (EJARCI) (FREIRE FILHO JR, et al., 2018). Um instrumento de medida que poderia contribuir para avaliar os acadêmicos quanto às suas experiências interprofissionais. Apesar da disponibilidade de instrumento validado, foi encontrado apenas um estudo na literatura nacional que a utilizou.

Segundo Freire Filho JR, et al. (2018) essa escala foi desenvolvida para mensuração das atitudes dos indivíduos envolvidos na colaboração interprofissional. Esse instrumento foi recentemente adaptado transculturalmente e validado no Brasil. Portanto, sugere-se a sua utilização em estudos com acadêmicos de odontologia para uma maior evidência científica. Os estudos selecionados apresentaram diferentes tipos de experiências interprofissionais: oito estudos descreveram experiências do Programa de Educação pelo Trabalho para a Saúde (PET-Saúde), três estudos abordaram a interprofissionalidade em um projeto de extensão e quatro estudos incorporaram a disciplina com inclinação para EIP na grade curricular do curso de odontologia.

Os resultados apontam a relevância do programa PET-Saúde, que influenciou a criação das disciplinas com abordagem interprofissional e também de projetos de extensão, observando-se a influência desse programa no âmbito da interprofissionalidade do curso de odontologia. Segundo Saraiva AM, et al. (2018) o PET-Saúde trouxe uma nova perspectiva sobre a EIP para as Escolas, nesse contexto os alunos tinham a possibilidade de uma aprendizagem ativa com análise crítica do funcionamento do SUS e das necessidades sociais dos usuários. Os acadêmicos tiveram a oportunidade de vivenciar a aplicação dos princípios e diretrizes do SUS, alinhados aos colegas de outras profissões da saúde, inseridos na interprofissionalidade, multiprofissionalidade, interdisciplinaridade e integralidade no cuidado à saúde.

Embora o PET-Saúde seja tão importante nas experiências dos estudos selecionados, há que se considerar que o programa apresenta suas limitações. O estudo de Pereira PM (2016) salienta a dificuldade de adesão de acadêmicos em muitas IES, já que a grade curricular por vezes não conta com "áreas verdes", os alunos cheios de atividades não conseguem participar do programa. Portanto, deve-se observar a 
importância de atividades extracurriculares como essa para a formação do cirurgião-dentista e rever a estrutura curricular do curso.

Os estudos selecionados aconteceram nos estados de Minas Gerais, Paraíba, Goiás, São Paulo, Paraná e Rio Grande do Sul. Um maior número de IES envolvidas com EIP no ensino odontológico foi observado nos estados de Minas Gerais e São Paulo (Figura 2).

Figura 2 - Distribuição por IES.

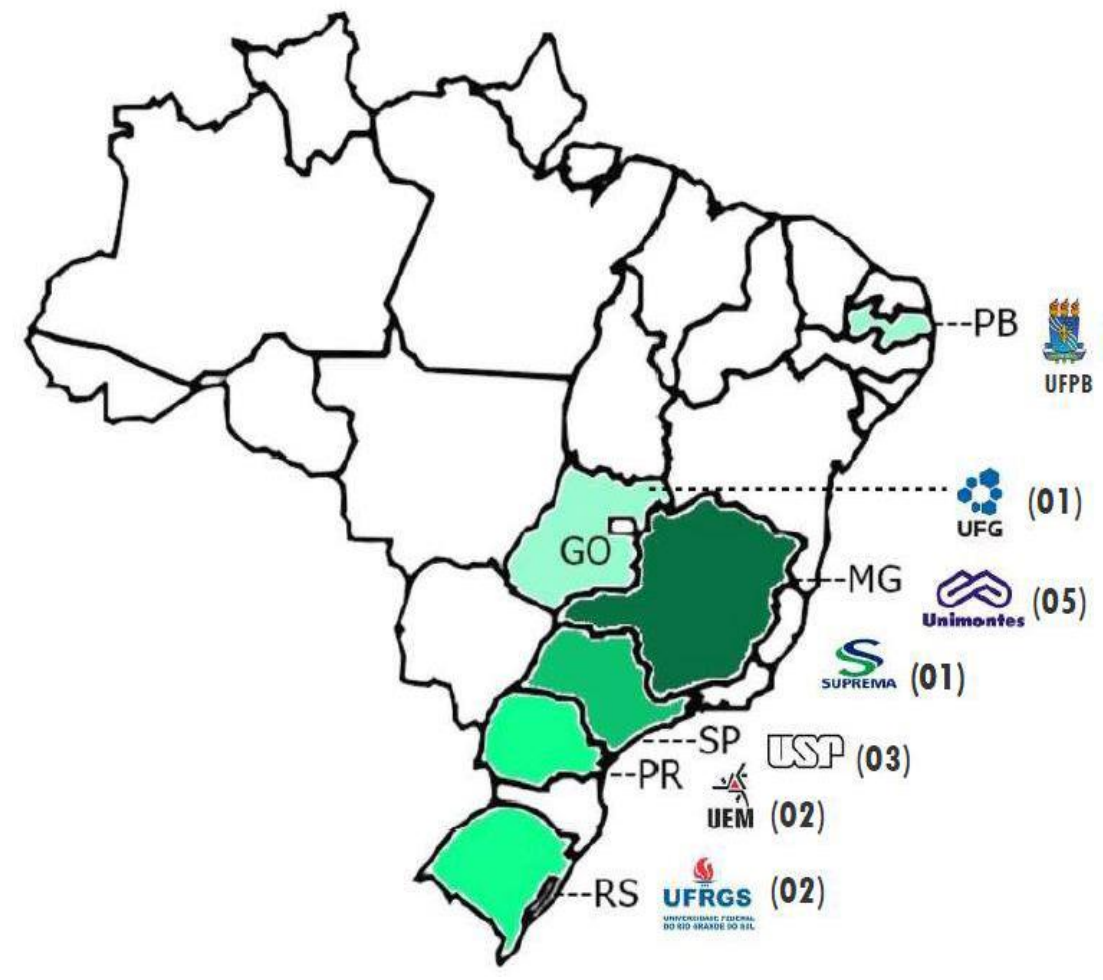

Fonte: Dias KDC, et al., 2021.

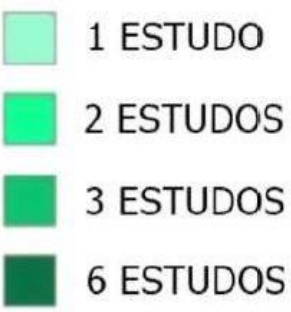

A Universidade Estadual de Montes Claros (Unimontes), localizada no norte de Minas Gerais, destaca-se por possuir cinco dos quinze trabalhos analisados. Todos esses estudos da Unimontes descrevem experiências exitosas do PET-Saúde, podendo-se constatar que o curso de odontologia se encontra $33,+$ alinhado aos fundamentos teóricos e práticos da EIP, desempenhando pioneirismo na região. Tais estudos caracterizam-se como resumos de relatos de experiência, diante disso sugere-se a ampliação para publicações de maior evidência científica.

Analisando a distribuição geográfica, percebe-se que a EIP tem permeado a odontologia de maneira heterogênea nas distintas regiões do Brasil, permitindo a reflexão sobre a necessidade de estratégias para uma distribuição mais uniforme da EIP no país. Em contraste a essa realidade brasileira, um estudo realizado nos Estados Unidos por Jiang T, et al. (2020) demonstrou 90\% (56/62) das escolas de odontologia norteamericanas oferecendo experiências interprofissionais aos seus alunos como parte integrante do currículo. Desde 2014 a odontologia nos Estados Unidos vem direcionando seus esforços para EIP, observando maior homogeneidade na distribuição geográfica.

Outro fator que se deve considerar é a predominância de instituições públicas nos estudos analisados, correspondendo a 93\%. Nota-se, assim, que as IES públicas estão mais engajadas no ensino interprofissional do que as IES privadas. Segundo Cassiano CCZ (2016), em estudo realizado na Universidade Federal de Goiás (UFG), os alunos que não eram bolsistas do PET-Saúde rejeitaram participar de algumas atividades que envolviam a EIP, sugerindo que a motivação dos acadêmicos esteja não apenas no aprendizado, mas também em demais benefícios do programa. 
Por fim, considerando o panorama brasileiro, que apresenta um consolidado sistema público de saúde, pode-se sugerir que este seja um fator facilitador para a implantação da EIP. Um estudo desenvolvido por Davis JN, et al. (2018) aponta maiores dificuldades de envolvimento com a EIP em países europeus nos quais os sistemas de saúde são predominantemente privados.

As unidades básicas de saúde se mostram como um cenário privilegiado para o desenvolvimento da EIP, presentes em $80 \%$ dos estudos analisados.

Os estudos de Cassiano CCZ (2016) e Fonseca GS, et al. (2017) relatam que os alunos reconhecem a importância do contato com a Unidade Básica de Saúde (UBS) desde os períodos iniciais, pois dessa forma se sentem mais capazes de compreender o real funcionamento do SUS e da Atenção Primária à Saúde (APS). Além de, nessa oportunidade, interagir com os outros profissionais e com os usuários.

Entretanto, é igualmente significante, abordar a EIP em hospitais, asilos e universidades. O estudo de Zajkowski LA, et al. (2017) mostrou como a interação interprofissional entre acadêmicos de odontologia e de terapia ocupacional mudaram a realidade de uma idosa em um asilo. Assim como o estudo de Fraga AT e Gislheni AP (2019) mostra também a atuação Interprofissional (IP) de acadêmicos de Fisioterapia, Medicina, Odontologia, Fonoaudiologia, Nutrição, Educação Física e Enfermagem num hospital atuando com idosos e garantindo cuidados mais integrais e estes.

Diante disso, é importante não estar limitado ao cenário da UBS, que pode ser a porta de entrada para a EIP na graduação, mas utilizar a EIP em todas as fases de graduação e nos mais diversos cenários de atuação.

Os aspectos relevantes para o êxito das experiências foram o planejamento das ações, organização, dedicação e persistência. Os trabalhos de Cavalcanti MS, et al. (2019), Zajkowski LA, et al. (2017), Fraga AT e Ghisleni AP (2019) demonstram ações pontuais planejadas e estritamente organizadas.

O estudo de Fonseca GS, et al. (2017) relata a experiência de uma acadêmica de odontologia que realizou uma observação participante em todos os setores de funcionamento da rotina da UBS. Para alcançar êxito nessa experiência, foi necessário persistência e dedicação, já que sua presença externa ao consultório incomodava, a saúde bucal era vista de forma muito fragmentada da saúde geral. Gradativamente a acadêmica tornou-se cada vez mais aceita e seu trabalho ganhou mais visibilidade.

No estudo Pereira PM (2016) os acadêmicos inseridos em uma experiência IP, uma disciplina prática baseada na EIP com grupos multiprofissionais fazendo trabalhos na UBS, quando questionados sobre 0 conceito da EIP não souberam responder. Diante disso, deve-se atentar ao construto teórico no qual se fundamentam as experiências IP, para que a experiência não perca seu sentido.

Nos estudos de Noronha FS, et al. (2019), Oliveira JL, et al. (2019) e Lima CR, et al. (2019) foram relatadas oficinas com os integrantes das ESF para capacitação sobre a importância da EIP. Esses trabalhos mostram a importância de toda a equipe estar alinhada num mesmo propósito em que o foco principal é à melhoria do serviço prestado.

Ademais, a EIP torna-se ainda mais efetiva quando os próprios pacientes conseguem compreender a atuação IP, conforme Silva MLO, et al. (2019). Neste trabalho houve uma oficina sobre IP com os pacientes e, assim, eles puderam compreender a importância de serem tratados por diversos profissionais concomitantemente.

Diante disso, a fim de se exercer plenamente a EIP, deve-se aprimorar resiliência, fundamentação teórica e romper paradigmas e barreiras estabelecidas pelo modelo flexneriano de saúde.

Através da análise dos quinze estudos pode-se perceber como EIP influenciou positivamente no ensino odontológico. Os estudos destacaram a troca de saberes, a valorização do papel de cada profissional, o respeito entre cada profissão, a desconstrução de estereótipos das outras profissões e o despertamento de novas habilidades e competências, consolidando a visão de integralidade em saúde e contribuindo para a melhoria dos serviços oferecidos à população (Figura 3). 
Figura 3 - Contribuições da EIP ao ensino odontológico.

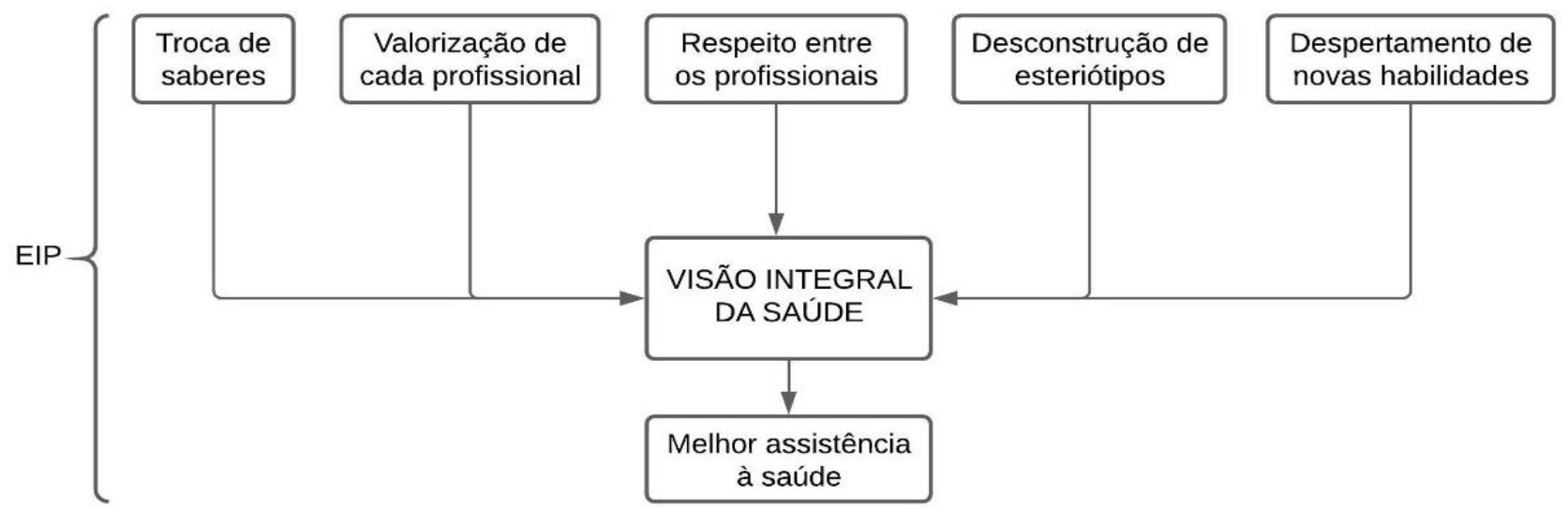

Fonte: Dias KDC, et al., 2021.

A experiência relatada no estudo de Cavalcanti MS, et al. (2019) descreve acadêmicos de medicina, enfermagem e odontologia realizando juntos a atividade de escovação supervisionada em escolares. Essa experiência aproxima as três profissões e desperta nos acadêmicos de medicina e enfermagem o desenvolvimento de novas habilidades que não seriam adquiridos sem a interação IP proporcionada. Tal aproximação é também identificada no estudo norte-americano de Okeke CC (2020), que aponta a aplicação da perspectiva odontológica em outras áreas da saúde e, concomitantemente a influência dessas áreas na odontologia.

Outra experiência relatada por Souza RMR, et al. (2013) mostra uma acadêmica de odontologia participando das consultas de puericultura com a enfermeira de uma UBS. O objetivo era contribuir com os ensinamentos de higienização bucal de bebês e aprender sobre a saúde geral dos bebês. Esse relato demonstra as trocas de saberes e suas implicações os envolvidos. Adicionalmente, verificam-se, aspectos importantes a serem desenvolvidos: respeito e a valorização de cada profissão. Lima CR, et al. (2019) identificaram em seus estudos uma ampla competição entre indivíduos avaliados, que se sentiam inseguros quanto a sua identidade profissional.

Diante do exposto, entende-se que aspectos citados são componentes essenciais da EIP. Em consonância com Reeves S (2016), essas premissas integram o fundamento da EIP: compartilhamento de responsabilidades entre duas ou mais profissões para aprimorar a qualidade da assistência.

\section{CONSIDERAÇÕES FINAIS}

Verificou-se que a maioria das produções científicas envolvendo acadêmicos de odontologia, enquadradas na temática deste estudo, consiste em relatos de experiência. Foi evidenciada expressiva atuação do setor público, considerando a influência do programa PET-Saúde nas experiências desenvolvidas em IES Estaduais e Federais, tendo como cenário principalmente as Unidades Básicas de Saúde. Constata-se, então, que a EIP parece influenciar positivamente o ensino odontológico no Brasil. Esta revisão sistemática encontrou um vasto campo a ser melhor explorado na literatura da odontologia. Através desse estudo não se pode pretender uma mudança curricular para os cursos de odontologia no Brasil, devido à maioria dos estudos analisados exigirem maior evidência científica. Esse fator se configura como a principal limitação desta revisão sistemática. Entretanto, espera-se que este trabalho desperte na comunidade odontológica o esforço crescente para investigação e aplicação da EIP.

\section{REFERÊNCIAS}

1. ALENCAR ES, ALMOULOUD SA. A metodologia de pesquisa: metassíntese qualitativa. Revista Reflexão e Ação, 2017; 25(3): 204-220.

2. ANDREWS EA. The Future of Interprofessional Education and Practice for Dentists and Dental Education. Journal of Dental Education, 2017; 81(8): 186-192. 
3. ARAÚJO TAM, et al. Multiprofissionalidade e interprofissionalidade em uma residência hospitalar: o olhar de residentes e preceptores. Interface, 2017; 21(62): 601-613.

4. CASSIANO CCZ. A prática dos estágios em Saúde Coletiva e PET-Saúde/RAS na formação em odontologia no Sistema Único de Saúde. Dissertação (Mestrado em Ensino em Saúde) - Faculdade de Medicina. Universidade Federal de Goiás, Goiânia, 2016; 135 p.

5. CAVALCANTI MS, et al. Prática de escovação supervisionada com crianças em escola de Montes Claros: Uma Atividade Interprofissional. Anais on-line do 13ํ FEPEG Unimontes, 2019.

6. CONSELHO FEDERAL DE ODONTOLOGIA. 2019. In: Ofício CFO 111/2019. Brasília: CFO Conselho Federal de Odontologia. Disponível em: https://website.cfo.org.br/. Acesso em: 22 jun. 2019.

7. DAVIS JN, et al. Interprofessional education in dental education: an international perpective. Eur J Dent Educ., 2018; 22(1): 10-16.

8. DE-LA-TORRE-UGARTE-GUANILO MC, et al. Revisão sistemática: noções gerais. Revista da Escola de Enfermagem da USP, 2011; 45(5): 1260-1266.

9. FARIAS DN, et al. Interdisciplinaridade e interprofissionalidade na estratégia de saúde da família. Trab. educ. saúde, 2018; 16(1): 141-162.

10. FERRAZ AMLJ, et al. Formação interprofissional em saúde: uma mudança de paradigma Curricular. Revista da ABENO, 2014; 14(2): 57.

11. FONSECA GS, et al. Da fragmentação à formação interprofissional: proposta de um modelo de estágio supervisionado para a graduação em odontologia. Saúde em Redes, 2017; 3(4): 410-424.

12. FRAGA AT, GHISLENI AP. Educação Interprofissional nas graduações de saúde em Gerontologia. Cad Edu Saúde e Fis, 2019; 6(12): 98-99.

13. FREIRE FILHO JR, et al. Atitudes para a colaboração interprofissional de equipes da Atenção Primária participantes do Programa Mais Médicos. Revista Latino-Americana de Enfermagem, 2018, 26 (e3018): 1-8.

14. FUKUI VI. Aprendizagem Baseada em Projeto: Unidade Básica de Saúde como cenário pedagógico interprofissional. Anais eletrônicos do $42^{\circ}$ Congresso Universitário Brasileiro de Odontologia da USP, 2018.

15. GALVÃO TF, et al. Principais itens para relatar Revisões sistemáticas e Meta-análises: A recomendação PRISMA. Epidemiol. Serv. Saúde, 2015; 24(2): 335-342.

16. HAMIL LM. Looking Back to Move Ahead: Interprofessional Education in Dental Education. Journal of Dental Education, 2017; 81(8): 74-80.

17. JIANG T, et al. Interprofessional education in dental schools: Results of a national survey. Journal of Interprofessional Education and Practice, 2020; 18: 1-7.

18. LIMA AFS, et al. Avaliação da educação interprofissional em saúde como componente curricular na perspectiva nos discentes da Universidade de Pernambuco. Rev Cadernos do Cuidado, 2019; 3: 40-53.

19. LIMA CR, et al. Uma abordagem sobre a interprofissionalidade em uma Unidade de Saúde da Família (USF) de Montes Claros-MG. Anais on-line do 13ํFㄹ

20. MAIMARIS W, et al. The Influence of health systems on hypertension awareness, treatment, and control: a systematic literature review. PLoS Medicine, 2013; 10(7): 1-17.

21. NORONHA FS, et al. Interprofissionalidade em saúde: um relato de experiência do grupo PET-Saúde da USF Antônio Pimenta. Anais on-line do 13ํFㄹ EG Unimontes, 2019.

22. OKEKE CC. The role of interprofessional education in dentistry: a student narrative. Health, Interprofessional Practice \& Education, 2020; 4(1): 1-4

23. OLIVEIRA JL, et al. Oficina "Interprofissionalidade Em saúde": relato de experiência. Anais on-line do 13ํFEPEG Unimontes, 2019.

24. OSF. 2020. In: Interprofessional Education in Brazil and it's Contributions to dental education: a Systematic Review Protocol. Charlottesville: Center for Open Science. Disponível em: https://osf.io/ma6fq/. Acesso em: 2 ago. 2020.

25. OUZZANI M, et al. Rayyan-a web and mobile app for systematic reviews. BioMed Central, 2016; 5(1): 1-10.

26. PARENTE RCM, et al. Relatos e série de casos na era da medicina baseada em evidência. Brazilian Journal os Videoendoscopic Surgery, 2012; 3: 67-70.

27. PEDUZZI M, et al. Educação interprofissional: formação de profissionais de saúde para o trabalho em equipe com foco nos usuários. Rev. esc. enferm. USP, 2013; 47(4): 977-983.

28. PEREIRA PM. A educação interprofissional e o Pró PET-Saúde USP-Capital 2012/2014: a percepção de tutores, preceptores e estudantes. Dissertação (Mestrado em Ciências da Saúde) - Faculdade de Odontologia, Universidade de São Paulo, São Paulo, 2016; 110 p.

29. REEVES S. Porque precisamos da educação interprofissional para um cuidado efetivo e seguro. Interface: Communication, Health, Education, 2016; 20(56): 185-196.

30. ROCHA NB, et al. Percepções de aprendizagem sobre a disciplina interprofissional em Odontologia. Revista da ABENO, 2017; 17(3): 41-54.

31. SARAIVA AM, et al. Disciplina interprofissional em saúde: avaliação de discentes de Odontologia. Revista da ABENO, 2018; 18(4): 3-13.

32. SILVA MLO, et al. Relato de experiência: oficina interprofissionalidade com usuários de uma estratégia saúde da família do norte de Minas Gerais. Anais on-line do 13ํFEPEG Unimontes, 2019.

33. SOUZA RMR, et al. Promoção da saúde bucal para mães e bebês na USF Nova Conquista - João Pessoa/ PB relato de experiência de um grupo tutorial PET-Saúde da Família e redes. Revista da ABENO, 2013; 13 (2): 50-57.

34. SOUZA SV, ROSSIT RAS. Dilemas e perspectivas dos recursos humanos em saúde no contexto da pandemia. ENF. FOCO, 2020; 11(1): 68-73.

35. TOMPSEN NN, et al. Educação interprofissional na graduação em Odontologia: experiências curriculares e disponibilidade dos estudantes. Revista Odontológica da UNESP; 2018, 47(5): 309-80.

36. WEINTRAUB JA. What should oral health professionals know in 2040: executive summary. Journal of Dental Education, 2017; 81(8): 1024-1032.

37. ZAJKOWSKI LA, et al. Memória e saúde bucal: uma intervenção interprofissional em idosa institucionalizada. Rev. Fac. Odontol, 2017; 58(1): 1-32. 